Telehealth Governance: An Essential Tool to Empower Today's Healthcare Leaders

Bryan Arkwright, Jeff Jones, Thomas Osborne, Guy Glorioso, John Russo, Jr.

Strong telehealth governance serves as the cornerstone for advancing a telehealth strategy by ensuring that the health system has the intentional leadership infrastructure to compete and excel in this fast-paced and transforming industry. ${ }^{1}$

\title{
A Conceptual Framework
}

Effective governance is the essential first step towards successful management. The former informs the latter to optimize value to the stakeholders. Paraphrasing the Financial Reporting Council, corporate (telehealth) governance should contribute to better company performance by helping a board discharge its duties in the best interests of stakeholders: executive leadership, management, staff, customers, patients, vendors, communities, and regulators, etc.

Good governance facilitates efficient, effective, and entrepreneurial management that can deliver value over the longer term. If ignored, the consequences may be vulnerability or poor performance (Financial Reporting Council, 2008).

The authors embrace a philosophical argument for governance, that emphasizes active risk management and resource management to ensure alignment between long- and short-term strategies. This is accomplished through leadership, accountability, and responsibility in accord with the organization's mission, vision, and values. Three key functions of telehealth governance are explored here: management, prioritization of services, and achieving return on investment (ROI). In addition, how long it takes to create governance and milestones that define progress over time are addressed.

\section{Telehealth Management Capability}

Telehealth management capability provides the organization with timely, thorough, relevant, and accurate information about the telehealth industry. No entity exists in 
isolation. Accordingly, the state of the telehealth market in which the organization operates, and how its telehealth strategies and programs support and strengthen the organization's overall strategic and financial plans are essential. Simply put, the intent is to support and champion key telehealth stakeholder's decisions.

From an implementation perspective, the "telehealth executive champion" and the "telehealth leader" play important roles. Although not governance per se, these positions are critical in discharging governance directives once risks and opportunities are calculated. Leading healthcare organizations commonly select the telehealth executive champion from the following officers:

- Chief Information Officer

- Chief Technology Officer

- Chief Executive Officer

- Chief Operating Officer

- Chief Medical Officer

- Chief Medical Information Officer

Regardless of who the telehealth executive champion is, it is imperative that this person ensure telehealth options are evaluated as part of the planning process and ensure appropriate resources are allocated within the organization.

The telehealth leader supports the telehealth executive champion. Leading healthcare organizations commonly select this individual from the following personnel:

- Senior Vice President, Chief Telehealth Officer

- Vice President, Telehealth

- Executive Director or Director, Telehealth

- Medical Director, Telehealth

- Administrator, Telehealth

- Manager, Telehealth 
The telehealth executive champion and key telehealth stakeholders regularly and systematically review the telehealth leader's contributions to the healthcare organization.

Recruitment of the telehealth leader can be challenging. Leading health systems commonly employ the following tactics when recruiting and retaining telehealth leaders:

- Internal hires who have supported or assisted telehealth planning or operational implementation within the organization

- Internal hires with experience in business development and leading clinical operations

- External hires with a proven experience starting and leading a matrix-aligned telehealth program

- Interim leadership from a vendor agnostic organization, with proven experience starting and leading a matrix-aligned telehealth program

Interim leadership expedites initiation and maturing of a telehealth program and ensures effective transfer of accountabilities and responsibilities once an internal or external hire is installed as the telehealth leader.

A top priority of the telehealth executive champion and the telehealth leader is formation a multi-disciplinary team of clinical and administrative leaders to serve on an executive and/or steering committee for telehealth. An example of the departments represented on a telehealth executive and/or steering committee includes:

- Business Development

- Clinical Engineering / Biomedical

- Clinical Operations

- Compliance

- Employee Health

- Finance

- Innovation

- IT / IS (information technology/information systems) 
- Legal

- Marketing

- Medical Staff / Medical Affairs

- Nursing

- Philanthropy

- Population Health

- Quality

- Revenue Cycle

The telehealth executive champion, executive and/or steering committee, and the telehealth leader establish policies and procedures for developing, operating, recruiting, and compensating all key telehealth stakeholders involved with telehealth. This include, but is not limited to:

- Clinical Providers

- Full and Part-time Support staff

- Medical Director Leadership Dedicated to Telehealth

Maintaining a succession plan for management is another top priority, and includes identifying telehealth clinical provider roles, technical roles, and administrative roles.

Examples of maintaining telehealth management capability include selecting leadership at executive and primary operation levels, forming an executive and/or steering committee, and frequently evaluating a dashboard (i.e., metrics and key performance indicators) of performance against plans according to operations, clinical, technical, and financial goals.

Telehealth committee effectiveness (derived from White and Griffith's Well Managed Healthcare Organization) meets seven key needs: ${ }^{2}$

1. Meet legal requirements (licensing across state and international lines, credentialing at facilities and payers, coding, billing, reimbursement, hardware and software, security, CMS (Centers for Medicare \& Medicaid Services), 
JCAHO (Joint Commission on Accreditation of Healthcare Organizations), and state/country specific departments of health and human services

2. Compliance, policies, and procedures that back-up and align with legal requirements

3. Continuing education

4. Use of dashboards and automated data tracking

5. Culture

6. Conflicts of interest

7. Telehealth ROI Performance (i.e., clinical, operational, financial, technical)

Figures 1 and 2 illustrate examples of organizational structures.

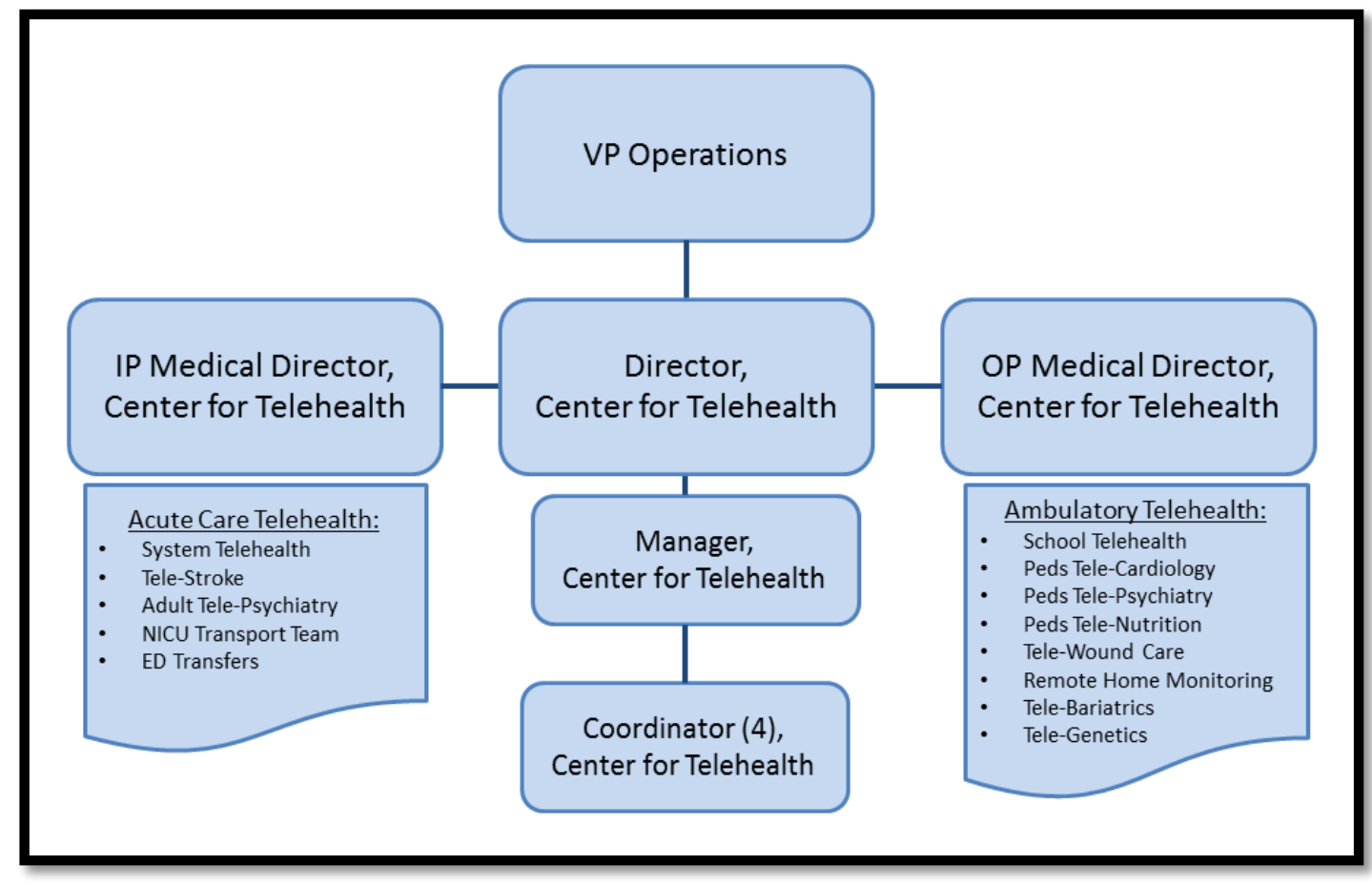

Figure 1. Organizational structure in which all department telehealth programs report to the Center for Telehealth Director and the VP of Operations. (Mediumsized eight (8) hospital health system in the Southeast). 


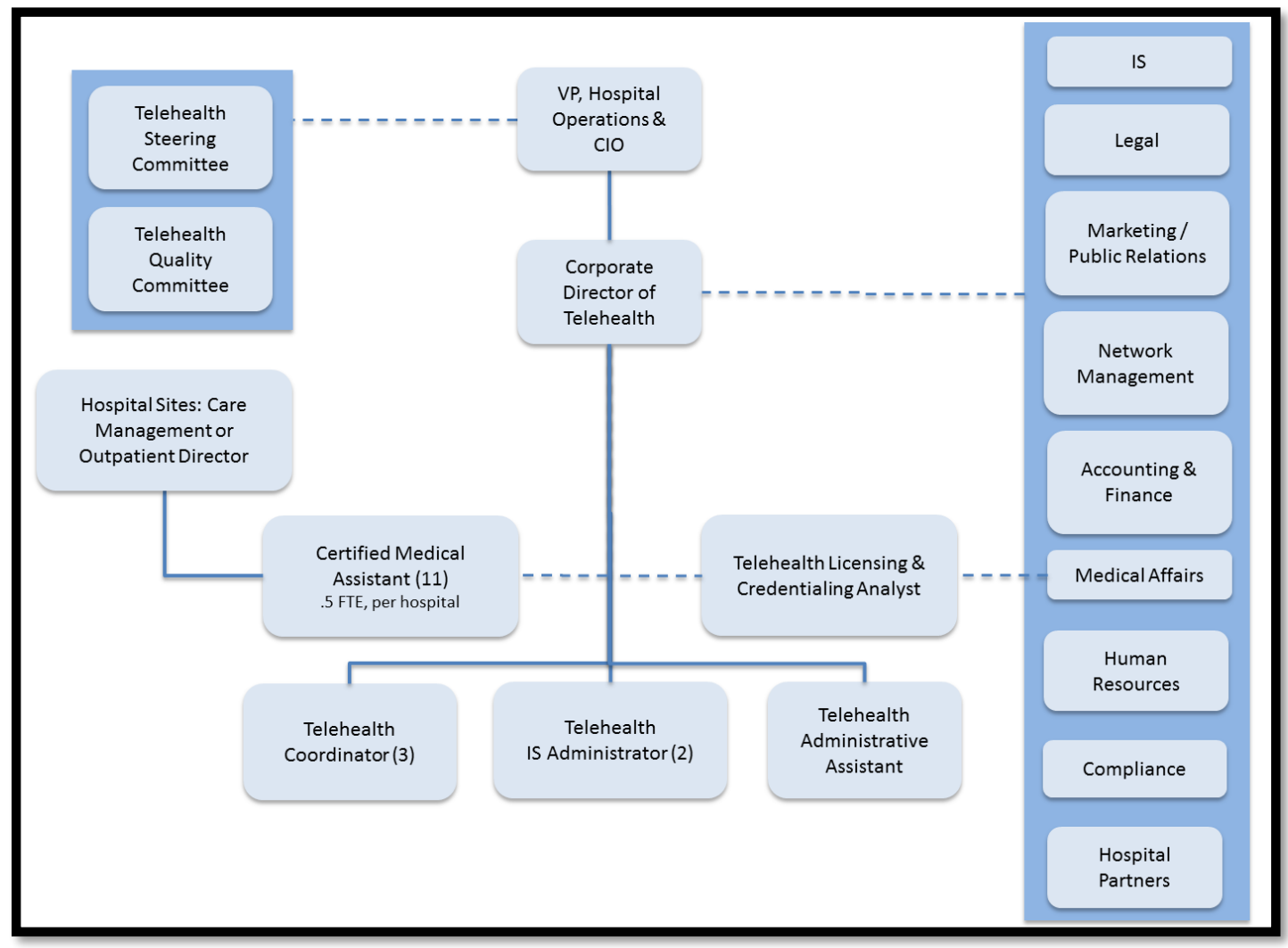

Figure 2. Organizational structure with shared reporting to either the VP, Hospital Operations \& CIO or the Corporate Director of Telehealth. Ultimately, all departments are under control of the VP, Hospital Operations \& CIO. (Largesized twenty plus [20+] hospital national health system).

\section{Using Telehealth Governance to Prioritize Services}

Ongoing systematic review and prioritization of telehealth services complements telehealth governance. This includes starting new programs, as well as prioritizing or vetoing recommendations to optimize and expand services. A proven approach is assessment, with consideration of a defined organizational telehealth methodology.

Telehealth methodology used by leading organizations may include the following:

- Clinical Value: Implementing the telehealth application significantly improves patient experience and access, while reducing cost and improving quality. 
- Physician/Provider Engagement: A physician or provider champion candidate is present with significant buy-in from colleagues in the clinical discipline. A strong team and team lead are present with a lead backup.

- Administrative Support: Senior leadership supports and validates the physician or provider champion and the clinical discipline's strength for successful implementation. Appropriate legal and risk counsel has been contacted.

- Strategic Plan Congruence: The clinical discipline and the telehealth application align with the organization's strategic plan.

- Access to Funding and Technology: The clinical discipline, or if present, telehealth office/department, has access to funding (federal grant, industry grant, foundation grant, association grant, organizational funding/capital, or other). Technology may exist or new technology investment may be required. The initiative's reimbursement is understood.

- Clinical Capacity: The clinical discipline has the capacity (i.e., time and manpower) for successful implementation short-term and long-term (1, 3, and 5 years). Implementing impacts capacity for the clinical discipline in a positive and manageable way.

- Operational and Logistical Complexity: Ease of implementation does not pose major operational barriers. Pre-work may or may not be accomplished to date.

Discussion of telehealth ideas or services against telehealth methodology helps determine if the organization is prepared for changes to telehealth services. The decision not to implement change, or to optimize or optimize and expand should consider existing telehealth services.

A systematic methodology defines and ranks readiness for the telehealth opportunity, ensuring that the organization doesn't make decisions for the sake of appearances or because a concept appears innovative. It assures that key stakeholders are on the same strategic page and helps prioritize and focus resources. Ideally, the telehealth application will score high on all factors listed above. However, reality often falls short of ideal. When it does, a clear methodology allows resources to focus on areas that 
require coaching or removal of barriers.

Process mapping of the proposed telehealth idea or service is a critical interactive whiteboard exercise for key clinical and administrative stakeholders. Ideally, it is facilitated by the telehealth leader experienced in change management, as well as gathering and process mapping key stakeholder comments, discussion, and input. Leading organizations map both the current state (no telehealth) of the clinical process, as well as the ideal state of the clinical process using telehealth.

Succinctly summarizing a telehealth idea or service against the methodology, along with process maps and all relevant data, is the last component for using telehealth governance to prioritize services. Summarized findings are presented to the telehealth executive and/or steering committee for approval. Key clinical and administrative stakeholders should attend the presentation by the telehealth executive/steering committee, and ensure they have reviewed and signed off on the summarized findings.

A recommended template for summarizing findings is an A3-inspired two-page work plan. Current and ideal process maps are depicted on one side of the form (Figure 3, 4), while the following elements are listed on the front page:

- Program Name

- Project Team

- Accountability

- Problem Statement

- Background Importance

- Baseline Data

- S.M.A.R.T. Statement (Specific, Measurable, Actionable, Realistic, and Timely) goal for the telehealth service

- Future State and Improvements

- Readiness Factor Notes and Score Assessment

- Implementation/Go Live Plan with Dates and Accountability

- Expected Outcomes 
- Cost and ROI Calculation

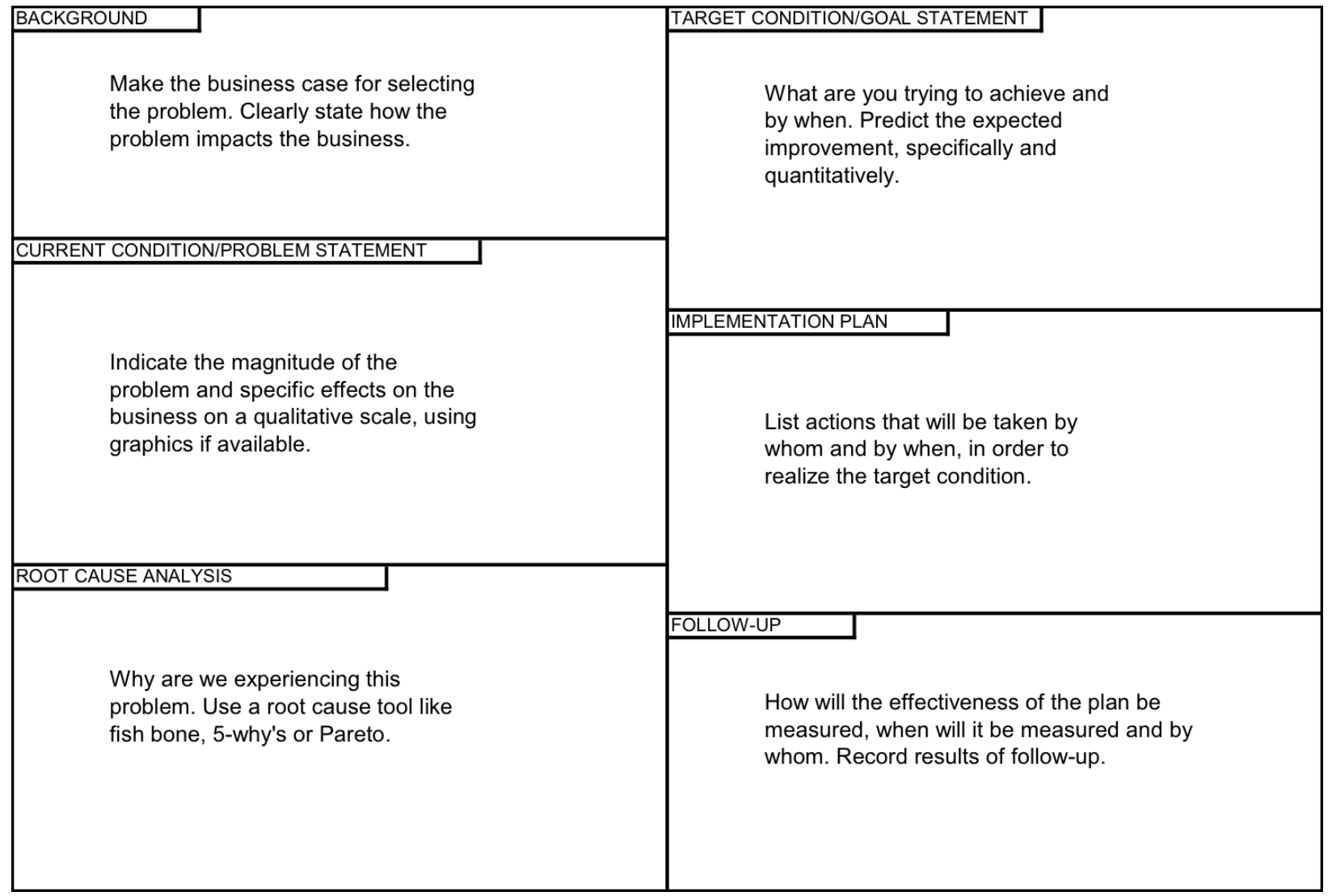

Figure 3. The A3 Report is a storyboard that includes essential steps in an improvement event, using one large sheet of paper. 


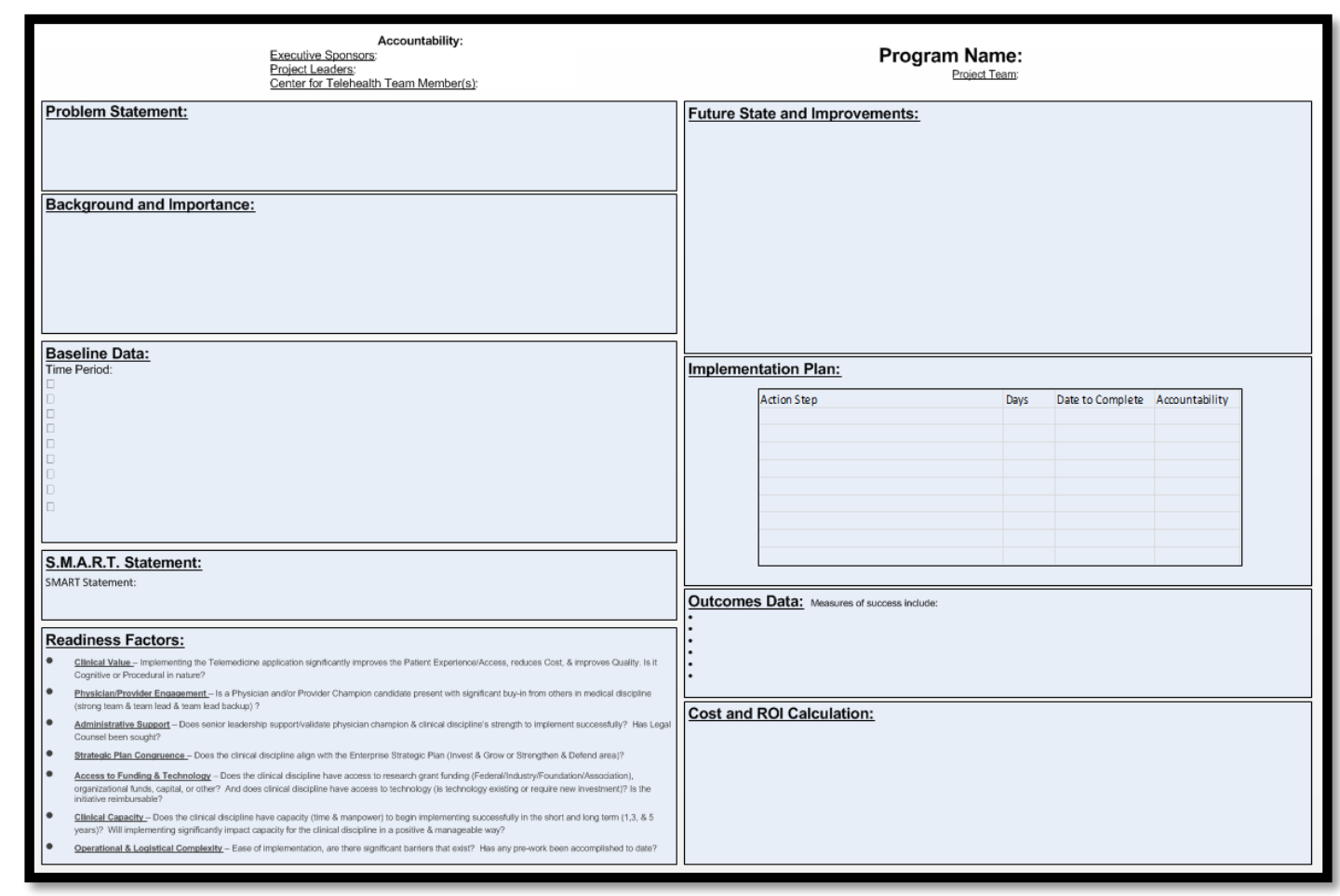

Figure 4. Example of A3 inspired blank template with organizational telehealth methodology.

\section{Using Telehealth Governance to Track Outcomes and Telehealth ROI}

"In any, case health care managers facing a decision must deal with the phenomenon known as bounded rationality, or the limits imposed on decision making by costs, human abilities and errors, time, technology, and the tractability of data," asserts Yasar Ozcan, Vice Chair and Director, Master of Science in Health Administration program at Virginia Commonwealth University. ${ }^{3}$

Once the approved telehealth service is implemented, monitor and track progress monthly against the summarized findings as presented to the telehealth executive/steering committee. Monitor progress and adjust expectations as needed. Reports are prepared quarterly, as well as annually and monthly. 
Review quarterly reports of the strategic telehealth $\mathrm{ROI}$ scorecard across clinical, financial, operational, and technical areas.

Telehealth ROI's are unique and complex for every organization according to the maturity of the telehealth program. The telehealth $\mathrm{ROI}$ includes indirect and direct variables; and telehealth-specific data can be challenging to collect across different healthcare organization information systems (e.g., EHR [electronic health records], video servers, telehealth vendor platforms, remote home monitoring platforms, HR (human resource) systems, financial systems, and more). Consider using automated software to facilitate rapid and ongoing tracking of telehealth $\mathrm{ROI}$.

\section{Annual Review}

The quarterly review facilitates annual reports on individual telehealth services and the collective telehealth full portfolio performance. The annual report ensures that the telehealth governance function tracks and communicates telehealth effectiveness as it relates to the overall healthcare organization's strategic goals/plan. Leading healthcare organizations that regularly track and communicate effectiveness create and ensure a strong telehealth culture that grows across the organization. Examples include a confidential survey of key telehealth stakeholder perspectives, as well as information from the quarterly review and monthly updates.

\section{Monthly Newsletter}

Similarly, a monthly internal telehealth email newsletter facilitates preparation of quarterly reports, as well as informing key stakeholders and clinical providers. Within the organization these communications perform a management and culture growth tactic. Leading healthcare organizations include the following elements in their newsletters:

- Monthly frequency

- Telehealth Program News and Updates

- Telehealth Business Intelligence/data on current program and specific service operations 
- Telehealth Market/Competitive Intelligence (links of relevant telehealth news/information within the market, which the organization operates)

- National/International Telehealth News and Trends

- Faculty/Provider Spotlights (editorial on new and emerging programs/leaders within the organization)

- Feedback and follow-up links to allow key stakeholders to share feedback on content and/or report news/information for the next month's update

\section{How Long to Establish Telehealth Governance?}

The time required to create and establish a telehealth governance program varies significantly among organizations. Some key questions include:

- Is there an existing governance structure that can be leveraged for the use of telehealth?

- What level of organizational experience with governance is available?

- Are telehealth outcomes closely measured, monitored, and reported today?

- Are there established telehealth success criteria?

- Do telehealth programs and services require a formal business plan-including an $\mathrm{ROI}$ - before they are approved? If yes, are results and savings measured and compared against the plan?

- Are other health informatics initiatives evaluated in terms of success and impact?

- Is there a clear leader and executive sponsor for the telehealth program and services?

- How engaged are physicians and providers in the telehealth services?

- Are there formal policies, procedures, and internal controls for other health informatics initiatives?

- Does a defined telehealth organizational structure exist?

- Are the telehealth services clearly defined and documented?

- Are there defined telehealth workflows?

- Is there a succession plan for key telehealth leadership? 
- Do end users know who to contact for telehealth support and technical questions?

Obviously, answering these questions better positions the organization for quicker design and deployment of telehealth governance.

\section{Milestones to Success}

A sound telehealth governance structure quickly pays dividends in proportion to the effort spent creating it. Milestones to success include:

- Physicians, providers, and patients are well-informed on the telehealth services and choices available to them

- Satisfaction with telehealth is high

- Telehealth outcomes are consistently measured and reported

- Telehealth successes and challenges are effectively reported

- Telehealth service scope, policies, procedures, and workflows are taught, known, and available to end users

- End users are trained on the use of telehealth

- Risks are mitigated

- Education and training is available and accessible to all

- The organization quickly adjusts to and updates process with regulatory changes or payments

- Clinical care quality is not impacted negatively by telehealth

\section{Summary}

The momentum and benefits of telehealth escalate with the organization's confidence to create a sustainable thriving and winning telehealth culture. Embrace telehealth governance as a tool to foster discussion and empower today's healthcare leaders and harness the transformative power of telehealth. 
1. Deloitte Consulting LLP. Empowering Patients with Telehealth. January 2016. URL: https://www2.deloitte.com/content/dam/Deloitte/us/Documents/publicsector/us-fed-empowering-patients-with-telehealth.pdf. Accessed 4/16/17.

2. Gheorghe M. Risk management in IT governance Framework. Economia. Seria Management, 14(2), 545- 552. URL:

https://www.google.com/url?sa=t\&rct=i\&q=\&esrc=s\&source=web\&cd=2\&cad=ria \&uact=8\&ved=0ahUKEwiP68L00bDTAhVLyoMKHc1NDKoQFggyMAE\&url=http \%3A\%2F\%2Fwww.management.ase.ro\%2Freveconomia\%2F20112\%2F27.pdf\&usg=AFQjCNHmPDeKx8 t1UVtl2pwV2-2dJMlgQ. Accessed 4/19/17.

3. White KR, Griffith, JR. The Well-Managed Healthcare Organization. $8^{\text {th }}$ edition. Health Administration Press. July 2, 2015. UR:\}L: https://www.amazon.com/WellManaged-Healthcare-Organization-

Eighth/dp/1567937217/ref=pd sbs $14 \mathrm{img} 0$ ? encoding=UTF8\&psc=1\&refRID =TB6AFPP2716JW1ZQKQ2Y. Accessed 4-16-17.

4. Ozcan, YA. Chapter 3; Decision Making in Health Care Facilities 2005, in Quantitative Methods in Health Care Management, Techniques and Applications. 2nd Edition. Jossey-Bass; $2^{\text {nd }}$ Edition, May 18, 2009. URL: https://www.amazon.com/Quantitative-Methods-Health-CareManagement/dp/0470434627/ref=sr 1 1?s=books\&ie=UTF8\&qid=1492367252 $\underline{\& r}=1-$

$1 \&$ keywords=Quantitative+Methods + in + Health + Care+Management $\% 2 \mathrm{C}+$ Techni ques+and+Applications. Accessed 4/16/17. 


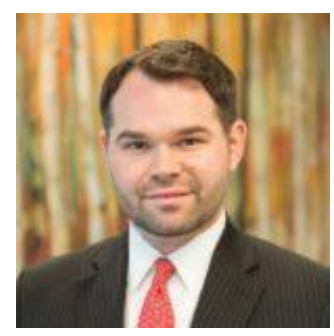

Bryan T. Arkwright works as a Managing Consultant with Schumacher Clinical Partners Consulting Services focusing on Telehealth / Telemedicine Strategy and Operations. He is an accomplished thought leader in the telehealth industry and is an avid speaker at regional and national healthcare conferences. In addition to Schumacher Clinical Partners, Bryan serves on the Editorial Board for the peer reviewed digital journal Telehealth and Medicine Today and is an Adjunct Faculty Member with the Wake Forest University School of Law. Bryan's additional areas of expertise include: Telehealth Startups, Project Management, Business Development, Digital Health, Strategic Planning, and Process Improvement. Bryan has a Bachelor's degree from Ohio University, a Master of Health Administration from Medical College of Virginia/VCU, and a Six Sigma Black Belt from the American Society for Quality.

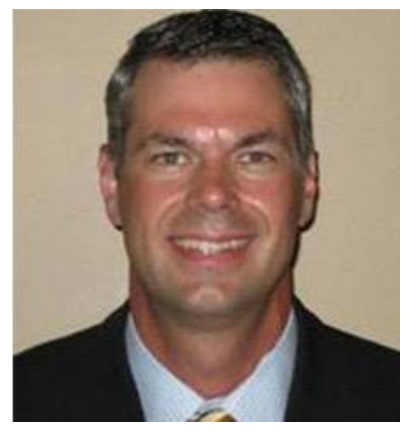

Jeff Jones is Director, Consulting Services at Schumacher Clinical Partners; Telehealth/Telemedicine Strategy \& Operations. 


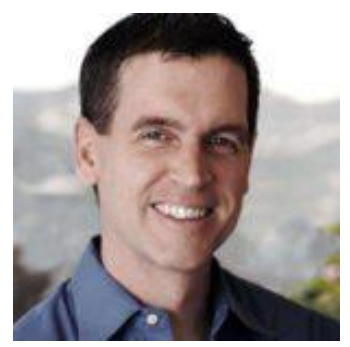

Thomas F. Osborne, MD, is Director of Medical Informatics at vRad (Virtual Radiologic), the leading teleradiology services and telemedicine company, which leverages its proprietary technology, data, and analytics capabilities to rapidly move patient images and information across a secure global network of 2,100 medical facilities in all 50 states.

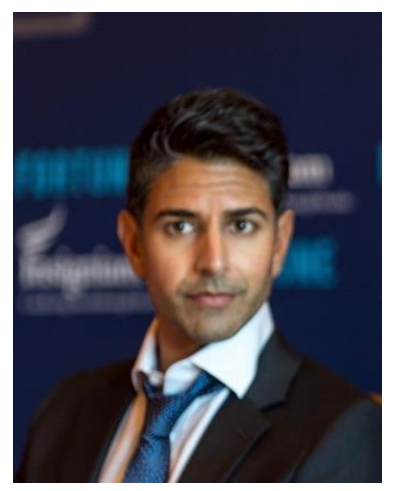

Guy Glorioso is Director, System Care Coordination, Virtual Care, Carolinas Healthcare System, Charlotte, North Carolina.

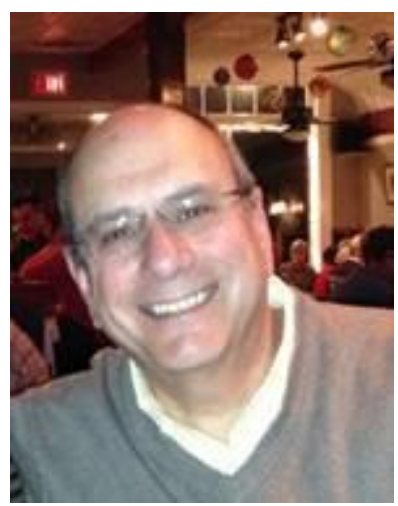

John Russo, Jr, PharmD, is Managing Editor of Telehealth and Medicine Today. 
Mr. Arkwright conceived the topic and wrote the manuscript. Mr. Jones, Dr. Osborne, and Mr. Glorioso provided guidance and technical overview. Dr. Russo, provided editing and organization support.

\section{Annotated Glossary}

\section{Telemedicine Governance:}

Finch T, May C, Mort M, Mair F. Telemedicine, telecare, and the future patient: innovation, risk and governance. In, New Technologies in Health Care. University of York. 2006. URL: https://link.springer.com/chapter/10.1057/9780230506046 7. Accessed 4/19/17.

This chapter charts the problematic interactions around a field of telecommunications in medicine, or, perhaps, a field of medicine in telecommunications. In it, the autors explore how 'telemedicine' has failed to normalize as part of everyday clinical practice in British health care, how attention has shifted first to a mode of practice which we call 'telehealthcare' and how this has been displaced by a new mode of practice-telecare.

May C, Mort M, Mair F, Finch T. Telemedicine and the 'future patient'? risk, governance and innovation. Summary of final Report. URL:

https://www.york.ac.uk/res/iht/projects/218252067/MayFinalRptSummaryRefs.pdf. Accessed 4/19/17.

Telemedicine and telehealthcare are justified by the presumed preferences of patients for faster access to local services, and that offer greater 'choice' about modes of access. Telehealthcare is seen to offer ways to achieve these priorities, and on this basis is presumed to be welcomed by patients and citizens. However, telemedicine and telehealthcare have implications for patients, and for their relationships with health professionals and the NHS, that go beyond issues of access, and the trade-offs that patients are willing to make against various aspects of health care services are assumed rather than known. 
Stanberry B. Legal ethical and risk issues in telemedicine. Computer Methods and Programs in Biomedicine. 2001;64 (3):225-33. URL:

http://www.cmpbjournal.com/article/S0169-2607(00)00142-5/abstract. Accessed 4/19/17.

This paper summarizes the main topics at a workshop hosted by the Centre for Law Ethics and Risk in Telemedicine at the 2nd International Conference on Advances in the Delivery of Care, held at the Institute of Health Sciences, City University, London, 1999. The workshop offered a unique opportunity for practitioners in the fields of telemedicine and shared care to discuss the legal, ethical and risk issues arising from the practice of medicine and healthcare from a distance using information and communication technologies ('ICTs') with a panel of experts from the fields of medicine, law, insurance and risk management.

Mort M, Finch T, May C. Making and Unmaking Telepatients:

Identity and Governance in New Health Technologies. Science, Technology, \& Human Vaues. 2009;34:9-33. URL:

http://journals.sagepub.com/doi/abs/10.1177/0162243907311274. Accessed 4/19/17. The authors examine how telehealthcare technologies assume certain forms of patients—or "telepatients" — who can be mobilized and combined with images and artifacts that speak for them in the clinical encounter. A tentative intervention is made in these emerging identities in the form of facilitating some alternative discourses about telehealthcare.

Mort, M, May, C, Finch, T, Mair, FS. Telemedicine and clinical governance: controlling technology, containing knowledge. In: Gray A. and Harrison, S. (eds.) Governing Medicine: Theory, Practice and Prospects. Open University Press: London, 2004. pp. 107-121. ISBN 9780335214358 http://eprints.gla.ac.uk/65062/

This book brings together a range of detailed explorations of the theory, practice, and prospects of clinical governance by some of the most eminent practitioners and researchers in the United Kingdom. Since New Labour's institution of clinical governance through its White Paper in 1997, there has been a good deal of debate 
about the history, theory and practice of clinical governance and the governance of clinical care. Divided into three parts, the book focuses on the core areas of: medicine, autonomy and governance; evidence, science and medicine; realizing clinical governance

Nelson R, Staggers N. Health Informatics - E-Book: An Interprofessional Approach. Elsevier Health Sciences. 2018. URL:

https://books.google.com/books?hl=en\&lr=\&id=eROwDQAAQBAJ\&oi=fnd\&pg=PP1\&dq =telehealth+governance \&ots=1zLy1kelr9\&sig=MIMCWO1kBKHh9iGJfgqSpVuH5Ns\#v= onepage\&q=telehealth\%20governance\&f=false. Accessed 4/19/17.

Health informatics is recognized as a disciple and a profession. Core disciplines include informatics, along with terminal competencies or learning outcomes, providing the framework for developing curricula within the healthcare professions. Learning outcomes include the skills, knowledge, and professional aptitudes expected of all graduates with the profession.

\section{IT Governance:}

George C. Whitehouse D, Duquernoy P. (eds) eHealth: Legal, Ethical and Governance Challenges. Springer. 2013. URL:

http://dl.acm.org/citation.cfm?doid=2728770.2656385. Accessed 4/19/17. Many directly transferrable ideas, references, frameworks and models can be adopted from fully developed "IT Governance" (this started getting serious around 1993) and Project Management publications.

\section{Corporate Governance:}

Smallwood RF. Chapter 10: Information governance and information technology functions. In, Information Governance: Concepts, Strategies, and Best Practices. Wiley. 2014. URL:

https://books.google.com/books?id=m5U6AwAAQBAJ\&pg=PT199\#v=onepage\&q\&f=fal se. Accessed 4/19/17.

This book is for ClOs, legal advisors, governance and compliance professionals, records managers, and anyone who is involved in managing content and information at 
an enterprise level. The field of information governance is about more than just transferring traditional records management strategies to new technologies. There are lessons learned from corporate governance failures in the 1980s and again in 20012002 with accounting fraud, and again with the financial crisis in 2008... This lead to adoption of some international standards. There's a lot of info out there on this.

Tommey M. The Infornomix Letter. 2008. URL:

http://www.infonomics.com.au/Web\%20Content/Documents/The Infonomics Letter Sp ecial Edition Nov 2008.pdf. Accessed 4/19/17.

Plain language about corporate governance of information technology.

Category: Policy \& Regulation

Tags:

Governance, organizational structure, telehealth, telemedicine process improvement, telemedicine program implementation 